\title{
Cloud Qos Ranking Prediction using Tanimoto Coefficient Similarity Based Deep Learning Method
}

\author{
S.S.Sujatha, S.Beghin Bose
}

\begin{abstract}
Cloud computing is a service which provides virtualized resources conforming to the end-user needs. Infrastructure, platform and software included in it. For the last two decades, it has achieved very gigantic growth. Currently, there are several cloud service providers in the market. The primary aim of this research is to minimize cloud service violation. It helps the service providers in exempting the penalty enhancing their reliability. So, cloud service $Q O S$ prediction is a research problem that must be solved. It is a very necessary thing for cloud service providers and cloud users. We have discussed several QoS prediction related to researches in the literature survey. But none of them has given a satisfactory QoS prediction. In this paper, we proposed a Tanimoto Coefficient Similarity-Based Deep Learning Method for QoS ranking prediction. The analysis helps service providers choose a suitable prediction method with optimal control parameters so that they can obtain accurate prediction results and avoid violation penalties. In comparison with the prior method in practice, the proposed method is more significant in terms of prediction accuracy, prediction time and error rate.
\end{abstract}

Keywords: Deep Learning, Artificial Intelligence, Ranking Prediction, Optimization, QoS, Cloud Computing.

\section{INTRODUCTION}

Cloud computing is a developing technology that is exponentially increasing the interest among users to use cloud applications [9]. Cloud computing is a model for enabling ubiquitous, convenient, on-demand network access to a shared pool of configurable computing resources that are rapidly provisioned and released with minimal management effort. Cloud services are the broad category that encompasses the many IT resources provided over the internet. Besides, it also describes the professional services that support the selection, deployment and ongoing management of many cloud-based resources. Cloud services are introduced to present easy, scalable access to applications, resources and services that are fully managed by the cloud services provider. Quality-of-Service (QoS)[10,11] is an essential idea for service selection and user satisfaction

Revised Manuscript Received on February 05, 2020.

* Correspondence Author

Dr.S.S.Sujatha, MCA, M.phil, PhD, Associate Professor, ST Hindu College (Manonmaniam Sundaranar University) Abishekapatti, Trinelveli,627012,Tamilnadu, India, sujaajai@gmail.com.

S.Beghin Bose, M,Sc(SE),Reg No:17213152271004 Full time Ph.D scholar in computer science, ST Hindu college, Nagercoil,India, Manonmaniam Sundaranar University, Abishekapatti, Trinelveli,627012,Tamilnadu,India, beghinbose.s@gmail.com.

(C) The Authors. Published by Blue Eyes Intelligence Engineering and Sciences Publication (BEIESP). This is an open access article under the CC BY-NC-ND license (http://creativecommons.org/licenses/by-nc-nd/4.0/) in cloud computing. QoS rankings present valuable information for optimal cloud service selection from a set of functionally equivalent service candidates. QoS ranking prediction is the process of predicting the top-ranked cloud services. But, the existing ranking prediction techniques failed to increase the prediction accuracy and to reduce the prediction time.

Cloud service's QoS in next period helps the user in the determination of most adaptable services. Also, there are various classic QoS prediction approaches which comprise of collaborative filtering technology Bayesian networks, case-based reasoning, and combinational prediction approach. However, the existing ranking prediction techniques failed to increase the prediction accuracy and to reduce the prediction time. In turn, to rectify these limitation found in cloud service, this paper is proposed novel cloud QoS ranking prediction using Deep Learning and Tanimoto Coefficient Similarity.

The rest of the paper is synchronized as Section 2 analyses the related work. Section 3 explains the proposed system methodologies. The experiments are conducted in Section 4. Finally, Section 5 concludes the paper and gives a list of future work.

\section{LITERATURE REVIEW}

In this section of the paper shows various types of QoS ranking prediction algorithms already proposed are briefly described.

Shuai Dinga et al proposes two algorithms for predicting cloud QoS: Multirank1 and Multirank2 [1]. Both of these algorithms improve accuracy and service diversity. According to this method, similar users first find out by kendall rank correlation coefficient. Secondly, the Preference function between users is calculated. Based on this method, the diversity of recommended services is computed by the Multi Rank1 algorithm. Finally, the missing QoS value is predicted using the multi-objective optimization method.

Hua Ma et al introduces a time-aware method for predicting cloud Quality of Services. The Interval Neutrosophic Set (INS) theory was used here to measure the reliability of cloud service [2]. Kendall Rank Correlation Coefficient (KRCC) is used here to find neighbouring users. New INS operators with theoretical proofs were introduced to calculate the degree and ranking values. Time-aware trustworthiness ranking prediction was invoked for creating a ranked service list. 


\section{Cloud Qos Ranking Prediction using Tanimoto Coefficient Similarity Based Deep Learning Method}

Jieming Zhu et al developed an adaptive matrix factorization(AMF) method for Online QoS Prediction [3]. This AMF method is inspired by the commonly used collaborative filtering techniques. Further, this proposed AMF method incorporates online learning, adaptive weights and Box-Cox transformation methods. When the AMF model is updated, Adaptive Weights is used here to manage step size. An accurate user does not move to a large extent consistent with an inexact service while inexact user requires to move lot regarding accurate service. The stochastic gradient descent algorithm failed to increase the performance of prediction.

K. Jayapriya et al proposed correlated QoS ranking algorithm for QoS Ranking Prediction. In this way, QoS ranking prediction is implemented using correlated QoS ranking algorithm and data smoothing technique. According to [4] Correlated Qos Ranking is used to find a similar user and QoS Data Smoothing technique was used here to pre-process a very noisy data-set. To evaluate the accuracy, six different types of correlated ranking algorithms have been developed and compared with already developed methods.

Dileep V K et al proposed Multi-Objective Particle Swarm Optimization Based Ranking System for cloud quality of service prediction [5]. This method consists of three modules: screening, optimal pseudo services and clustering process. Based on this method, the appropriate candidate's services are selected through the screening module. Besides, candidate services are optimized by a multi-objective particle swarm optimization algorithm. Finally, the best services are selected through the hierarchical clustering algorithm.

According to the literature survey, various service violation has been detected. Our chief aim is to provide the service that is mentioned in the Service Level Agreement (SLA) to the cloud users from $n$ users and $m$ service through a good QoS prediction method and thereby reducing the QoS violation of the cloud service providers multiple times. For this, first, find out the similar user and similar services

\section{PROPOSED METHODOLOGY}

In this research, plan to develop a Tanimoto Coefficient Similarity-based Deep Learning Networks Ranking Prediction Model for Cloud Service for better prediction accuracy and minimum ranking time. In this model, the user who requests for the ranking prediction is termed as an active user. Tanimoto coefficient calculates the degree of similarity between the two samples. Tanimoto Coefficient Similarity Measure is used to find the similarity between the active user ' $u$ ' Then, top-k users who are similar to user ' $u$ ' are neighbor users Te values of similarity measures usually range from 0 to 1 . Where 0 represents a non-similar user and 1 represents a similar user. The architecture of the proposed system is shown in fig 1.

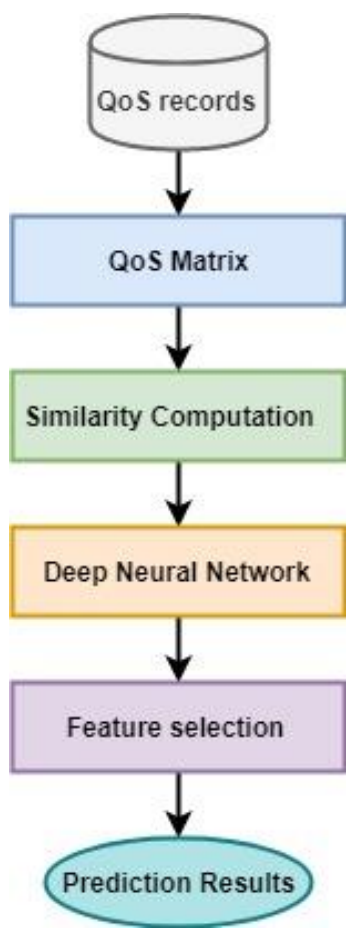

Fig 1: Architecture of Proposed Method

\section{A. QoS Matrix}

The QoS values are in a three-dimensional matrix format. Its architecture is shown in Fig. 2. M number of users $\mathrm{U}=\{\mathrm{U} 1, \mathrm{U} 2, \mathrm{U} 3---\mathrm{Um}\}$ using $n$ number of services $\mathrm{S}=\{\mathrm{S} 1, \mathrm{~S} 2$, $\mathrm{S} 3$...Sn). In this QoS Matrix, QoS values such as response time, throughput, latency, etc $\mathrm{Q}=\{\mathrm{Q} 1, \mathrm{Q} 2, \mathrm{Q} 3 \ldots \mathrm{Qn}\}$ used by users U.

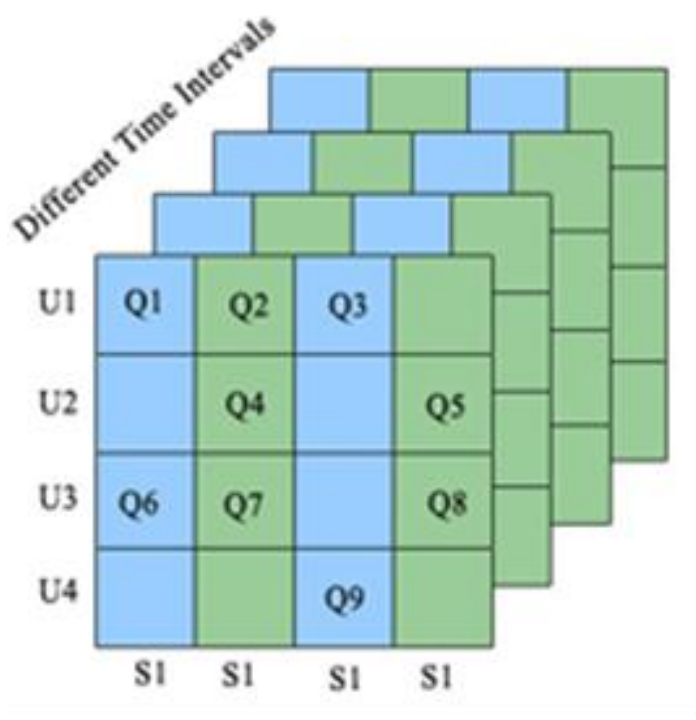

Fig.2. Architecture three-dimensional QoS matrix

\section{B. Similarity computation}

In some case, each attribute is binary such that each bit represents the absence of presence of a characteristic, thus, it is better to determine the similarity via the overlap, or intersection, of the sets. Simply put, the Tanimoto Coefficient uses the ratio of the intersecting set to the union set as the measure of similarity. Represented as a mathematical equation. 
Through the similarity computation, we can find similar cloud users and similar cloud services. To find the similar user Su use formula 1.

$$
S u=\frac{|U|}{|U i|}
$$

In this, $\mathrm{U}$ denote the total number of users. Ui denotes that the number of users $U$ are involved in service $i$.

$$
S i=\frac{\|I\|}{\|I u\|}
$$

Formula 2 is used to find out similar service Si. In this I represents the total number of services. Iu denotes the service that is invoked by user $\mathrm{u}$.

\section{Deep Learning Method}

Deep Learning Method [7][8] is based on the human brain. It is a soft computing technique that can solve the problems that cannot be solved by man. It has three important layers: input layer, hidden layer and output layer. Through the input layer, we provide input to Deep Learning Method. The hidden layer is placed between the input layer and the output layer. The Deep Learning Method provides the result to the users through the output layer. Its architecture is shown in Fig 3 .

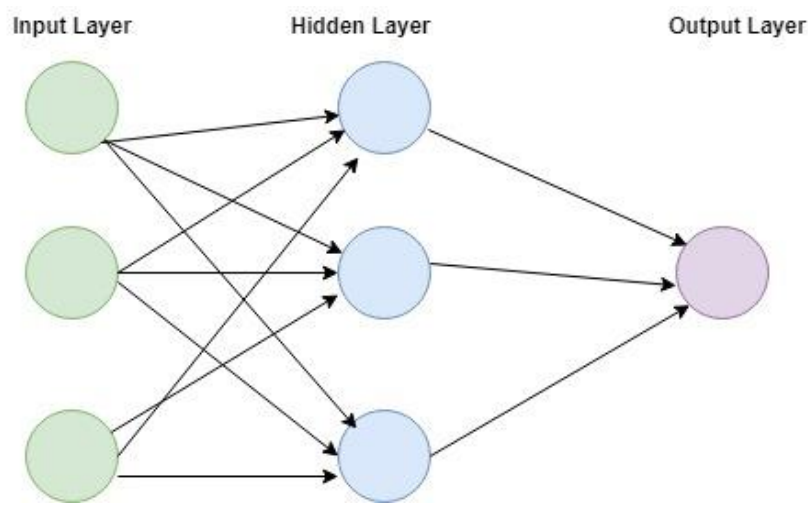

Fig.3. Structure of Deep Learning Methods

\section{Feature selection and learning}

Learning is an important process of Deep Learning Method. In our proposed method we take features from already used historical QoS data set and train them. For this purpose, we use the WSDream dataset. It is an open-source QoS dataset. It has been developed primarily for cloud QoS research. There are 5825 services and 329 users in this dataset. Moreover, two types of QoS metrics information's are available. E.g. Response time and throughput. Using formula 1 and 2 we find similar users and similar services and train them to the Deep Learning Method model. K-fold cross-validation method is used to test the proposed QoS prediction model. In this experiment, we test the proposed method in the ratio of 10:1. E.g. 90\% of data is used for training and the remaining is used for testing. By this way, the top-ranked user's cloud service gets predicted with minimal time consumption and higher accuracy. Experimental evaluation is carried out on factors such as prediction accuracy, prediction time and error rate with respect to the number of historical cloud user service.

\section{EXPERIMENTAL ANALYSIS}

The performance of the proposed Deep Learning-based QoS prediction method for Cloud services is evaluated by the following experiments.

\section{A. Dataset and Experiment Configuration}

The Cloud platform used in this paper is under windows operating system. WEKA [6] open-source AI tool is used for implementing this ranking prediction model. Moreover, Java JDK 1.8 is used as a software development environment, Eclipse IDE as an integrated development environment, used Tomcat 8.0 as a web server. The accuracy parameters of ANN are used for experimental analysis: accuracy, prediction time and error rate.

Accuracy: Accuracy is one metric for evaluating classification models. Formula 3 is used to find out the classification accuracy.

$$
\text { Accuracy }=\frac{\text { Number of correct predictions }}{\text { Total number of prediction }}
$$

Prediction time: The classifier takes the time to predict the QoS value. It is measured in milliseconds (ms).

Error rate: The classification error $\mathrm{Ei}$ of an individual program i depends on the number of samples incorrectly classified and is evaluated by the formula 4 .

$$
E i=\frac{f}{n}(4)
$$

Where $f$ is the number of sample cases incorrectly classified, and $\mathrm{n}$ is the total number of sample cases.

Table1 performance comparison by accuracy

\begin{tabular}{|l|l|}
\hline Method & Accuracy (\%) \\
\hline Proposed Method & 88 \\
\hline Multi-objective [1] & 82 \\
\hline Time-aware trustworthiness [2] & 84.3 \\
\hline Adaptive Matrix Factorization [3] & 85.9 \\
\hline Correlated QoS ranking algorithm [4] & 79.6 \\
\hline $\begin{array}{l}\text { Multi-Objective Particle Swarm Optimization } \\
\text { [5] }\end{array}$ & 75.4 \\
\hline
\end{tabular}

$$
\text { Accuracy Chart }
$$

Multi-Objective Particle Swarm Optimization [5]

$$
\text { Correlated Q0S ranking algorithm [4] }
$$
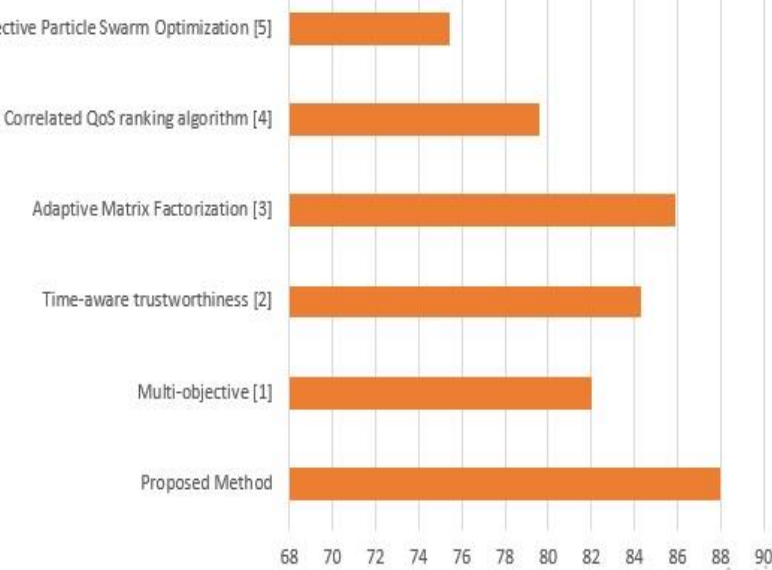

Fig.4. Accuracy chart 


\section{Cloud Qos Ranking Prediction using Tanimoto Coefficient Similarity Based Deep Learning Method}

Table1 shows the accuracy of the proposed method and the state of art algorithms. Fig4 shows the accuracy chart. It shows that the proposed Deep Learning Method for QoS Ranking Prediction methods achieve better accuracy than the existing methods.

Table2 Error rate

\begin{tabular}{|l|l|}
\hline Method & Error Rate (\%) \\
\hline Proposed Method & 12 \\
\hline Multi-objective [1] & 18 \\
\hline $\begin{array}{l}\text { Time-aware trustworthiness } \\
{[2]}\end{array}$ & 15 \\
\hline $\begin{array}{l}\text { Adaptive Matrix } \\
\text { Factorization [3] }\end{array}$ & 14 \\
\hline $\begin{array}{l}\text { Correlated QoS ranking } \\
\text { algorithm [4] }\end{array}$ & 42 \\
\hline $\begin{array}{l}\text { Multi-Objective Particle } \\
\text { Swarm Optimization [5] }\end{array}$ & 26 \\
\hline
\end{tabular}

Error Rate Chart

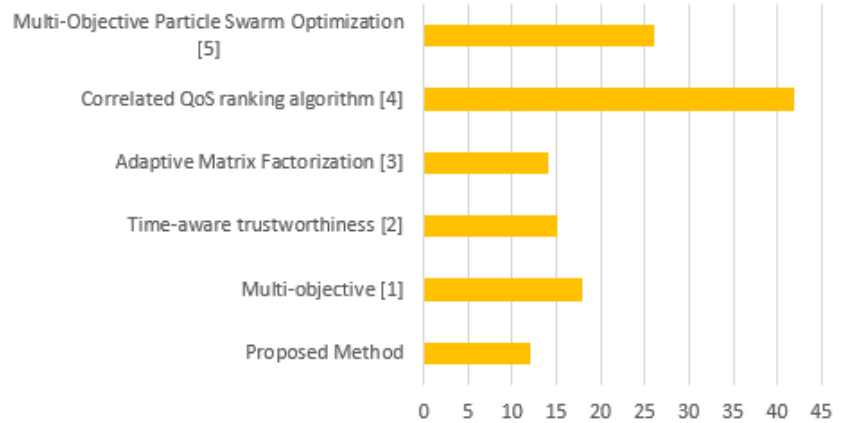

Fig.5. Error rate chart

Table 2 and fig5 shows the error rate of the proposed method and the state of art algorithms. It shows that the proposed Deep Learning Method for QoS Ranking Prediction method reduces the error rate multiple times than existing QoS Ranking Prediction methods.

Table 3 Prediction time

\begin{tabular}{|l|l|}
\hline Method & Prediction Time(ms) \\
\hline Proposed Method & 0.12 \\
\hline Multi-objective [1] & 0.34 \\
\hline Time-aware trustworthiness [2] & 0.37 \\
\hline $\begin{array}{l}\text { Adaptive Matrix Factorization } \\
\text { [3] }\end{array}$ & 0.39 \\
\hline $\begin{array}{l}\text { Correlated QoS ranking } \\
\text { algorithm [4] }\end{array}$ & 0.39 \\
\hline $\begin{array}{l}\text { Multi-Objective Particle Swarm } \\
\text { Optimization [5] }\end{array}$ & 0.41 \\
\hline
\end{tabular}

Retrieval Number: B4427129219/2020@BEIESP

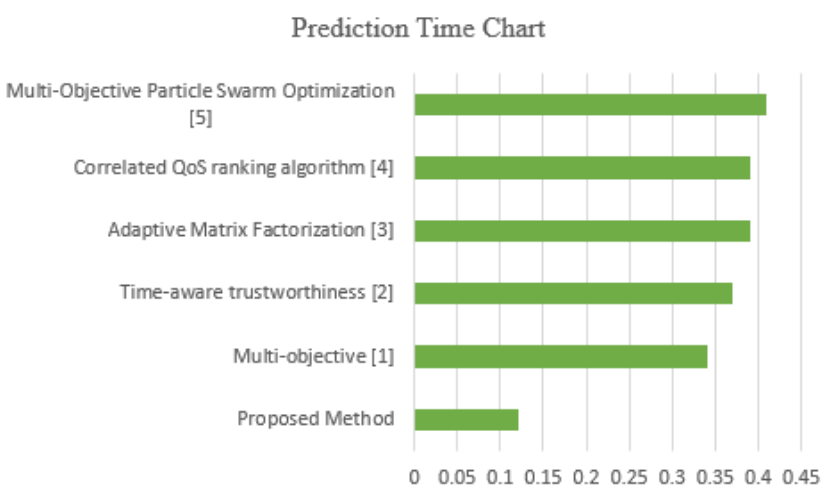

Fig.6. Prediction time chart

Table 3 and fig 6 shows the average QoS prediction time for the proposed QoS Ranking Prediction method and the existing methods. It shows that the Deep Learning Method reduces the QoS prediction time multiple times.

\section{CONCLUSION}

With its extensive features offered, cloud computing draws the need of the next generation in computing model. Due to its significance in science and commercial computing, it is widely utilized in academia and industry. The Quality of Service (QoS) is the most predominant factor among the selection of varied cloud services. Here, the paper describes all about a personalized QoS ranking prediction framework for cloud services where the proposed method is based on Deep Learning Networks and Tanimoto Coefficient Similarity. Also, the obtained results are analyzed with its prediction accuracy, prediction time and error rate. In spite of this system precise optimal ranking prediction with low error rate. Henceforth, the experiment results in demonstrating the efficiency of the system over the prior four approaches with the rate of higher accuracy.

\section{REFERENCES}

1. Shuai Ding et al, "Multi-objective Optimization based Ranking Prediction for Cloud Service Recommendation", Decision Suppor Systems, Elsevier, Volume 101, September 2017, Pages 106-114.

2. Hua Ma, Haibin Zhu, Zhigang Hu, Keqin Li and Wensheng Tang, "Time-aware trustworthiness ranking prediction for cloud services using interval neutrosophic set and ELECTRE", Knowledge-Based Systems, Elsevier, Volume 138, December 2017, Pages 27-45.

3. Jieming Zhu, Pinjia He, Zibin Zheng and Michael R. Lyu, "Online QoS Prediction for Runtime Service Adaptation via Adaptive Matrix Factorization", IEEE Transactions on Parallel and Distributed Systems, Volume 28, Issue 10, October 2017, Pages 2911 - 2924.

4. K. Jayapriya, "Cloud Service Recommendation Based on a Correlated QoS Ranking Prediction", Journal of Network and Systems Management, volume 24.

5. Dileep V K et al," An Efficient Multi-Objective Particle Swarm Optimization Based Ranking System for Cloud Service Selection", IET Communications, Volume: 13, Issue: 3, 2192019

6. Anand Kishor Pandey et al, "A comparative study of classification techniques by utilizing WEKA", 2016 International Conference on Signal Processing and Communication (ICSC). J. Jones. (1991, May 10). Networks (2nd ed.) [Online]. Available: http://www.atm.com.

7. Xuedan Du et al, "Overview of deep learning", 2016 31st Youth Academic Annual Conference of Chinese Association of Automation (YAC).

8. Ajay Shrestha et al, "Review of Deep Learning Algorithms and Architectures", IEEE Access ( Volume: 7 ).
Published By:

Blue Eyes Intelligence Engineering 
9. Maximilian Ahrens, "Cloud Computing and the Impact on Enterprise IT”, Future Internet Symposium FIS 2010: Future Internet - FIS 2010, Pages 148-155.

10. Mariem Jelassi et al, "A survey on quality of service in cloud computing”, IEEE 2017 3rd International Conference on Frontiers of Signal Processing (ICFSP).

11. Jolly Upadhyaya et al, "Quality of service in cloud computing in higher education: A critical survey and innovative model", 2017 International Conference on I-SMAC (IoT in Social, Mobile, Analytics and Cloud) (I-SMAC)

\section{AUTHORS PROFILE}

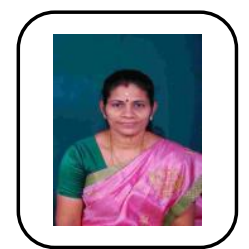

Dr.S.S Sujatha, in 1993 completed her MCA degree in Alagappa University, Karaikudi, and did her M.Phil degree in Manonmanium Sundaranar University, Tirunelveli during 2003. From 1994 onwards, she is working as an Associate Professor in the Department of Computer Science at S.T.Hindu College, Nagercoil . She completed her doctorate degree at Mother Teresa University, in 2014. Her thrust area is Image Processing, Cloud Computing and Data Mining.

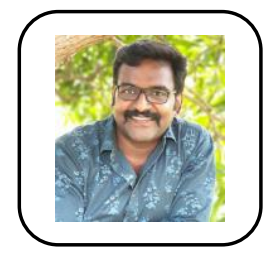

S.Beghin Bose is the Researcher in Manonmaniam Sundaranar University Tirunelveli. He did his post graduate degree, M.Sc Software Engineering in Manonmaniam Sundaranar University, Tirunelveli in 2007. He has a teaching experience of five years as an Assistant Professor in Narayanaguru Engineering College, Manjalumoodu from 2011 to 2016. At present he is doing his research as a full-time scholar in Hindu College of Arts and Science, Nagercoil. He is an active participant in various seminars and conferences. He also published several articles in the same. His research focus comprises of Cloud Computing, Big Data, Data Mining and Networking.. 